\title{
Massive AdS supergravitons and holography
}

\author{
Constantin Bachas \\ Laboratoire de Physique de l'École Normale Supérieure, \\ CNRS, Paris Sciences Lettres, Sorbonne and Paris-Diderot Universities, \\ 24 rue Lhomond, 75231 Paris Cedex, France \\ E-mail: bachas@physique.ens.fr
}

ABSTRACT: I compare two holographic mechanisms giving to the graviton a parametricallysmall supersymmetric mass $m_{g}$ in Anti-de Sitter spacetime. In the context of bimetric gravity these mechanisms couple 'weakly' two initially decoupled superconformal theories by: (i) turning on a double-trace deformation, or (ii) gauging a common global symmetry. Superconformal invariance restricts the number of Poincaré supercharges to $N_{Q} \leq 4$ for mechanism (i) and to $N_{Q} \leq 8$ for mechanism (ii), and the AdS dimension to $D \leq 5$. The putative effective supergravities are expected to break down in the $m_{g} \rightarrow 0$ limit at an intermediate scale between $m_{g}$ and $m_{\text {Planck }}$. In a recently-proposed microscopic embedding of mechanism (ii) in string theory $[7,8]$, I show that $m_{g}=0$ is at infinite distance in moduli space, and I relate the breakdown of the effective theory to the condensation of unprotected spin-2 excitations in the string-theoretic description of the 'holographic bridge'. The phenomenon is invisible in the weakly-coupled CFT side. I conjecture that similar phenomena should be at work in all cases.

KEYwords: AdS-CFT Correspondence, Superstring Vacua

ARXiv EPrint: 1905.05039 


\section{Contents}

1 Introduction 1

$2 \quad$ No go for $D>5$ and for $N_{Q}>8 \quad 3$

3 Holographic BEH mechanisms 5

3.1 Gauging versus double-trace deformations 5

$\begin{array}{lll}3.2 & \text { No multitrace for } N_{Q}>4 & 7\end{array}$

3.3 Stueckelberg multiplets 8

4 On massive AdS supergravities $\quad 10$

$\begin{array}{lll}4.1 & \text { Breakdown and a distance conjecture } & 10\end{array}$

4.2 Janus throat and the $\Lambda_{*}$ scale 11

\section{Introduction}

There has been considerable interest in recent years in relativistic theories of massive gravity, see e.g. [1-3] for reviews. The model-independent upper bound on the graviton mass $^{1}$ from the LIGO/Virgo collaboration [4] is $m_{g} \lesssim 7.7 \times 10^{-23} \mathrm{eV}$, which translates to a lower bound on its Compton wavelength $\lambda_{g} \gtrsim 1.5 \times 10^{13} \mathrm{~km}$ (or about 1.5 light years). It leaves ample room for a tiny but finite graviton mass with dramatic implications for the large-scale structure of the Universe.

An important question is whether effective low-energy theories of massive gravity have a consistent ultraviolet completion, or whether they are in the quantum-gravity 'swampland'. A direct embedding of massive Anti-de Sitter (AdS) gravity in string theory was recently proposed in refs. [7, 8]. Besides living in $\mathrm{AdS}_{4}$, this embedding has $\mathcal{N}=4$ supersymmetries and is clearly not realistic. It does, however, allow to address the breakdown of the effective massive-gravity theory in a controlled setup. Indeed, a common feature of all effective theories of massive gravity is that their range of validity vanishes when $m_{g} \rightarrow 0$, as follows by inspection of the non-linear action of the Stueckelberg field [9]. In $\mathrm{AdS}_{4}$ the breakdown scale is at most $\Lambda_{*} \sim\left(m_{g} m_{\mathrm{Pl}} / l_{\mathrm{AdS}}\right)^{1 / 3}$, where $l_{\mathrm{AdS}}$ is the AdS radius and $m_{\mathrm{Pl}}$ is the four-dimensional Planck scale [10]. In this letter I will identify the origin of this breakdown in the string-theory embedding of $[7,8]$.

A second question that I will address is whether similar string theory embeddings exist in other dimensions and with different amounts of supersymmetry. It is conceptually simpler to consider the more general bimetric theories, from which massive gravity can be obtained as a limit [1-3]. Assuming that all AdS vacua have holographic duals, one is led to study the following setup: two initially decoupled conformal theories are made to interact 'weakly' in a sense to be made more precise below. After coupling, the two conserved energy-momentum tensors mix so that their sum remains conserved while an orthogonal

\footnotetext{
${ }^{1}$ This bound comes from the dispersion relation. For other bounds on $m_{g}$ see $[5,6]$.
} 
combination acquires a small anomalous dimension $\epsilon_{g}$. This is the CFT description of two decoupled metrics interacting weakly, so that one combination obtains a mass while the second stays massless. The interaction of the conformal theories can be one of two kinds:

- A 'double trace' deformation $\int \phi \Phi$, with $\phi$ and $\Phi$ operators of the separate theories;

- Coupling through a messenger field, such as the gauging of a common global symmetry.

Note that the second mechanism differs from the first since massless messengers cannot be integrated out to give local double-trace interactions.

These mechanisms are constrained by the requirement of superconformal symmetry $(\mathfrak{g})$. The massless supergraviton is dual to a conserved energy-momentum tensor that belongs to a short representation of $\mathfrak{g}$. Higgsing combines it with a Stueckelberg supermultiplet into a long representation with anomalous scaling dimension $\epsilon_{g}$, where $\epsilon_{g} \sim m_{g}^{2}$ is parametrically small. The list of all unitary superconformal representations is given in ref. [11]. One sees by inspection that for SCFTs in more than four dimensions, or with more than half-maximal supersymmetry, the massless spin-2 multiplets are absolutely protected, i.e. they cannot combine to form long representations. The corresponding massive supergravities are thus a priori ruled out. The double-trace mechanism is further constrained by the requirement that the bridging operator be of product form, which is impossible with more than $\frac{1}{4}$-maximal supersymmetry. These bounds are saturated, as one can show with examples. Furthermore in all the allowed cases the Stueckelberg multiplets are made out of the ingredients of the 'bridge', namely (i) the operators $\phi$ and $\Phi$, or (ii) currents and free vector multiplets. This is a consistency check for these mechanisms.

Double-trace CFT deformations are described by modified boundary conditions in $\operatorname{AdS}_{D}[12,13]$, but their lift to string theory (or indeed simply to ten dimensions) is problematic. They were studied as a mechanism for Higgsing gravity in refs. [14-16], following up on the original proposal by Porrati [17] in the single-metric limit. The gauging mechanism in $[7,8]$ shares many features of [the AdS version of] Randall-Sundrum models [18-20]. As the first microscopic implementation of these ideas on the gravity side, it will allow us to study the breakdown of the putative effective theory. I will argue that the full stringtheoretic description of the bridge (in terms of a cutoff $\mathrm{AdS}_{5}$ Janus throat) is essential for the understanding of the $m_{g} \rightarrow 0$ limit.

An outstanding question is whether similar microscopic resolutions exist for all the other cases of holographic graviton Higgsing. Another interesting problem is the construction of massive supergravities, in particular half-maximal ones in $\mathrm{AdS}_{4}$ and $\mathrm{AdS}_{5}{ }^{2}{ }^{2}$ These theories should be highly constrained and could be compared with the ghost-free bosonic action of de Rham et al. [22-25]. ${ }^{3}$ I hope to return to these questions in the near future.

\footnotetext{
${ }^{2} \mathrm{~A} \mathcal{N}=4$ supergravity with four-derivative terms and a massive spin-2 mode has been constructed in ref. [21]. Here I refer however to standard two-derivative actions.

${ }^{3}$ There have been lingering concerns about pathologies of massive-gravity actions, see e.g. refs. [26, 27]. These arise, in essence, because one tries to make two disjoint metrics interact via an effective local action. The microscopic description of the interaction given in $[7,8]$ supports the view that, at least perturbatively around AdS spacetime, these concerns can be laid to rest.
} 
The plan of this paper is as follows. In section 2 I explain why massive Anti-de Sitter supergravities with more than half-maximal supersymmetry, as well as the cases $\mathcal{N}=1$ in $\mathrm{AdS}_{6}$ and $\mathrm{AdS}_{7}$, are ruled out. In section $3 \mathrm{I}$ describe the two holographic mechanisms for the superconformal Higgsing of the graviton, and argue that only $\frac{1}{4}$-maximal supersymmetry is possible in the double-trace case. Supersymmetry plays a minor role in most of the literature on massive gravity. But by guaranteeing the stability of AdS vacua and of their weak Higgsing interaction, it is bound to be of great help in any effort to embedd massive gravity in string-theory.

The reader not interested in the supersymmetric details can skip altogether subsections 3.2 and 3.3. Section 4 contains the main message of the paper and can be read independently. There I trace the breakdown of the effective theory when $m_{g} \rightarrow 0$ to towers of spin-2 modes in the string-theoretic description of the Higgsing mechanism proposed in $[7,8]$. The bridge is in this case a cutoff $\mathrm{AdS}_{5}$ Janus throat connecting two $\mathrm{AdS}_{4}$ vacua. Although the background supersymmetry is important for stabilizing the entire setup, the condensing spin-2 modes are unprotected and only visible on the string-theory side. I conjecture that similar phenomena might explain the breakdown in all other cases as well.

\section{No go for $D>5$ and for $N_{Q}>8$}

We are interested in $\mathrm{AdS}_{D}$ solutions of string theory around which the metric fluctuations have a mass, and the dimensionless parameter $m_{g} l_{\text {AdS }}$ can be tuned arbitrarily close to zero. Here $l_{\mathrm{AdS}}$ is the radius of $\mathrm{AdS}_{D}$, and $m_{g}$ is the graviton mass defined so that for the pure Einstein theory with cosmological constant $m_{g}=0$. The mass need not be a continuous parameter, all we assume is that it can be made parametrically small.

Field excitations in $\mathrm{AdS}_{D}$ are in unitary representations of the conformal algebra $\mathfrak{s o}(2, D)$. If the solution is supersymmetric the field supermultiplets are assigned to representations of the relevant superconformal algebra $\mathfrak{g} \supset \mathfrak{s o}(2, D)$. A massless spin-2 particle is in a short representation of the algebra. To obtain mass it must combine with a representation that provides the missing polarizations of the massive multiplet. The resulting representation is long, and its mass is unrestricted. Schematically

$$
\text { [Massive/Long] } \underset{m_{g} \rightarrow 0}{\longrightarrow}[\text { Massless/Short } \oplus \oplus[\text { Stueckelberg }]
$$

Now for many superalgebras the massless supergraviton is absolutely protected, i.e. it never appears in the decomposition of a long multiplet. When this is the case the graviton cannot obtain a mass without breaking some of the supersymmetries. In such cases the existence of AdS supergravity with tunable graviton mass can be excluded.

I restrict the discussion to the range $4 \leq D \leq 7$, or equivalently to the range $3 \leq d \leq 6$ where $d$ is the dimension of the dual SCFT. There are no superconformal theories in higher dimensions [28], while in $D<4$ there is no dynamical graviton. ${ }^{4}$ All unitary superconformal representations in this range have been listed in ref. [11]. The cases in

\footnotetext{
${ }^{4}$ The case of $\mathrm{AdS}_{3}$ is nevertheless very interesting, for massive $D=3$ supergravities see e.g. refs. [29, 30]. Three dimensions is special for many reasons, in particular because the conformal group is infinitely extended.
} 


\begin{tabular}{|c|c|c|c|}
\cline { 2 - 4 } \multicolumn{1}{c|}{} & Susy & $\mathfrak{g}$ & Massless graviton \\
\hline \multirow{2}{*}{$\operatorname{AdS}_{7}$} & $\mathcal{N}=(2,0)$ & $\mathfrak{o s p}\left(8^{*} \mid 4\right)$ & $D_{1}[0,0,0]_{4}^{(0,2)}$ \\
\cline { 2 - 4 } & $\mathcal{N}=(1,0)$ & $\mathfrak{o s p}\left(8^{*} \mid 2\right)$ & $B_{3}[0,0,0]_{4}^{(0)}$ \\
\hline $\operatorname{AdS}_{6}$ & $\mathcal{N}=1$ & $\mathfrak{f}(4)$ & $B_{2}[0,0]_{3}^{(0)}$ \\
\hline \multirow{2}{*}{$\operatorname{AdS}_{5}$} & $\mathcal{N}=4$ & $\mathfrak{p s u}(2,2 \mid 4)$ & $B_{1} \bar{B}_{1}[0 ; 0]_{2}^{(2,0,2)}$ \\
\cline { 2 - 4 } & $\mathcal{N}=3$ & $\mathfrak{s u}(2,2 \mid 3)$ & $B_{1} \bar{B}_{1}[0 ; 0]_{2}^{(1,1 ; 0)}$ \\
\hline \multirow{3}{*}{$\operatorname{AdS}_{4}$} & $\mathcal{N}=8$ & $\mathfrak{o s p}(8 \mid 4)$ & $B_{1}[0]_{1}^{(0,0,0,2)}$ or $(0,0,2,0)$ \\
\cline { 2 - 4 } & $\mathcal{N}=7$ & $\mathfrak{o} \mathfrak{p}(7 \mid 4)$ & $B_{1}[0]_{1}^{(0,0,2)}$ \\
\cline { 2 - 4 } & $\mathcal{N}=6$ & $\mathfrak{o} \mathfrak{p} p(6 \mid 4)$ & $B_{1}[0]_{1}^{(0,1,1)}$ \\
\cline { 2 - 4 } & $\mathcal{N}=5$ & $\mathfrak{o s p}(5 \mid 4)$ & $B_{1}[0]_{1}^{(1,0)}$ \\
\hline
\end{tabular}

Table 1. The $\operatorname{AdS}_{D}$ supergravities for which the massless graviton is in an absolutely protected representation. We list the number of supersymmetries, the superconformal algebra $\mathfrak{g}$, and the absolutely protected representation in the notation of ref. [11]. The list includes all cases in $D>5$ dimensions, and all cases with more than half-maximal supersymmetry for reasons explained in the main text. The table has redundancies: when the massless graviton is protected for given $\left(D, \mathcal{N}_{0}\right)$ it is also protected for all $\left(D, \mathcal{N}>\mathcal{N}_{0}\right)$.

which the massless supergraviton is absolutely protected are given in table 1 . I have adopted here the unifying notation of [11] in which a representation is denoted by the Dynkin labels of the superconformal primary, the letters $A$ or $B, C \cdots$ indicate whether a short representation is at the unitarity threshold or separated from the continuum by a gap, and the subscript gives the level of the first null states. Since the reader might not be familiar with this notation, I will translate to more standard ones where appropriate.

The multiplet of the massless AdS graviton, or of the dual conserved energy-momentum tensor, is always in a short representation of $\mathfrak{g}$. This is an $A$-type representation when Higgsing is allowed (see section 3 ) and a gapped, $B$ - or $D$-type, representation in all excluded cases. Note that the gap does not guarantee absolute protection of a generic multiplet. It is a necessary, not a sufficient condition.

The forbidden list of table 1 includes all cases with more than half-maximal supersymmetry, i.e. with $N_{Q}>8$ Poincaré supercharges, as advertized. The half-maximal cases in $D=6,7$ dimensions are also excluded. ${ }^{5}$ This ties in nicely with the fact that holographic mechanisms for Higgsing the supergraviton are not available for these $(D, \mathcal{N})$ pairs, as will be clear in the following section. A quick mnemonic for string theorists is that Higgsing is only possible if the dual $\mathrm{SCFT}_{d}$ can couple as a defect to a higher-dimensional bulk theory without breaking its own superconformal symmetries. This is indeed impossible whenever $N_{Q}>8$ or for $d=5,6 .^{6}$

\footnotetext{
${ }^{5}$ For $D=6$ (i.e. for $\mathrm{SCFT}_{5}$ ) maximal supersymmetry is not compatible with conformal invariance.

${ }^{6}$ Our counting of supersymmetries in the table is the standard counting on the field theory side, i.e. $\mathcal{N}$ is the number of spinor supercharges of the $\mathrm{SCFT}_{d}$. Superconformal symmetries double this number on the gravity side. The two maximal massive supergravities in particular, dual to $\mathcal{N}=4 \mathrm{SCFT}_{3}$ and $\mathcal{N}=2$ $\mathrm{SCFT}_{4}$, correspond to $\mathrm{AdS}_{D}$ vacua of gauged $N=4$ supergravity in $D=4$ and $D=5$ dimensions. In both cases the number of Poincaré supercharges of the SCFT is $N_{Q}=8$.
} 
Let me stress however that these no go statements are purely kinematical and do not depend on any details of the Brout-Englert-Higgs (BEH) mechanism. The only assumptions are superconformal invariance and unitarity.

It should be also noted that the existence of marginal superconformal deformations is neither a necessary nor a sufficient condition for the Higgsing of the supergraviton. Indeed superconformal manifolds exist for $\mathcal{N}=1,2,4$ in $d=4$ but only for $\mathcal{N} \leq 2$ in $d=3$ [31]. On the other hand, Higgsing of the supergraviton is forbidden for $\mathcal{N}=4$ in $\mathrm{AdS}_{5}$ whereas it is possible for $\mathcal{N}=4$ in $\mathrm{AdS}_{4}$.

\section{Holographic BEH mechanisms}

Let us take now a closer look at the mechanism by which the graviton obtains a mass. The most convenient starting point is a bimetric theory with effective action

$$
S=\frac{m_{\mathrm{Pl}}^{D-2}}{2} \int \sqrt{g}\left(R[g]+\mathcal{L}_{\mathrm{m}}\right)+\frac{M_{\mathrm{Pl}}^{D-2}}{2} \int \sqrt{G}\left(R[G]+\mathcal{L}_{\mathrm{m}}^{\prime}\right)+S_{\mathrm{int}}(g, G),
$$

where $g$ and $G$ are two independent metrics. Each of them has its own Einstein action with negative cosmological constant, and they couple minimally to separate matter fields whose Lagrangians are $\mathcal{L}_{\mathrm{m}}$ and $\mathcal{L}_{\mathrm{m}}^{\prime}$. In the absence of the interaction, $S$ describes two decoupled Universes. We assume that they both have CFT duals and work at leading order in $S_{\text {int }}$. At this order, $S_{\text {int }}$ does not affect the classical backgrounds $g_{0}$ and $G_{0}$, but it mixes the two metric fluctuations $g-g_{0}$ and $G-G_{0}$ making one linear combination massive. The limit $M_{\mathrm{Pl}} \rightarrow \infty$ decouples the massless mode leaving a theory of a single massive graviton.

\subsection{Gauging versus double-trace deformations}

The holographic viewpoint of this setup is as follows. The non-interacting Universes are dual to decoupled conformal field theories, $\mathrm{cft}_{d}$ and $\mathrm{CFT}_{d}$, whose energy-momentum tensors, $t_{a b}$ and $T_{a b}$, are separately conserved - they are dual to the two massless gravitons. The 2-point function of $t_{a b}$ [normalized by canonical Ward identities] reads

$$
\left\langle t_{a b}(x) t_{c e}(0)\right\rangle=\frac{c / 2}{x^{2 d}}\left(I_{a c}(x) I_{b e}(x)+I_{a e}(x) I_{b c}(x)-\frac{2}{d} \eta_{a b} \eta_{c e}\right),
$$

where $I_{a b}(x)=\eta_{b a}-2 x_{a} x_{b} / x^{2}$ and the central charge $c$ is related to the radius of the Anti-de Sitter background (whose metric is $g_{0}$ ) by the relation [15]

$$
c=\left(m_{\mathrm{Pl}} l_{\mathrm{AdS}}\right)^{d-1} \frac{d(d+1) \Gamma(d)}{2 \pi^{d / 2}(d-1) \Gamma\left(\frac{d}{2}\right)} .
$$

Similar expressions hold for $t \rightarrow T, c \rightarrow C, m_{\mathrm{Pl}} \rightarrow M_{\mathrm{Pl}}$ and $l_{\mathrm{AdS}}$ replaced by $L_{\mathrm{AdS}}$, the radius of the second AdS metric $G_{0}$.

Before turning on an interaction, both energy-momentum tensors have canonical scaling dimension $[t]=[T]=d$. The interaction splits this degeneracy. This is a problem of second-order degenerate perturbation theory, with the extra input that since the total energy-momentum tensor is still conserved its scaling dimension is unchanged. This 
determines at leading order in the perturbing Hamiltonian the two orthonormal energy eigenstates. They correspond (by the operator-state correspondence) to the two spin-2 operators

$$
\mathcal{T}_{a b}=\frac{1}{\sqrt{c+C}}\left(t_{a b}+T_{a b}\right)+O(\lambda) \quad \text { and } \quad \tilde{\mathcal{T}}_{a b}=\frac{1}{\sqrt{C c^{2}+c C^{2}}}\left(C t_{a b}-c T_{a b}\right)+O(\lambda),
$$

where $\lambda$ is the small parameter of the perturbation. Clearly, the conserved energymomentum tensor has canonical dimension $[\mathcal{T}]=d$ and is dual to a massless graviton, whereas the other, orthogonal combination acquires an anomalous dimension $[\tilde{\mathcal{T}}]=d+\epsilon_{g}$ and is dual in AdS to a massive spin-2 particle. $^{7}$

For CFTs coupled by a marginal double-trace operator, $\epsilon_{g}$ was computed in ref. [15] with the following leading-order result

$$
\epsilon_{g}=\lambda^{2}\left(\frac{1}{c}+\frac{1}{C}\right)+O\left(\lambda^{3}\right)
$$

Numerical factors that depend on the perturbing operator and the dimension $d$ (but not on the central charges) have been absorbed in the coupling $\lambda$. One sees that the decoupling limit $M_{\mathrm{Pl}} \rightarrow \infty$, which freezes the fluctuations of the auxiliary metric $G$ in massive-gravity theories, amounts to sending $C \rightarrow \infty$ and hence $\mathcal{T}_{a b} \rightarrow 0$ and $\tilde{\mathcal{T}}_{a b} \rightarrow \frac{1}{\sqrt{c}} t_{a b}$. This leaves a single massive spin-2 operator as expected.

So a bimetric theory is dual in holography to two conformal field theories that are weakly coupled to each other. But what does 'weak coupling' really mean from the gravity side? We want the two conserved spin-2 operators to be replaced by one conserved operator and another that acquires a very small anomalous dimension. Intuitively, this can happen not only if the interaction Hamiltonian is tunably small, but also if it involves a very small fraction of the CFT degrees of freedom. With this in mind we distinguish two holographic mechanisms for coupling the two Universes:

- Double-trace $\int \phi \Phi$, where $\phi$ is an operator of $\mathrm{cft}_{d}$ and $\Phi$ of $\mathrm{CFT}_{d}$;

- Mediation by messengers, such as in the gauging of a common global symmetry.

To preserve the $\operatorname{AdS}_{D}$ isometries these couplings must be marginal, or if they are relevant we follow them to an infrared fixed point. Note that since massive messenger fields can be integrated out to give multitrace couplings, the second mechanism is distinct from the first only if (some of) the messengers are massless.

Let us be a little more precise about the meaning of eq. (3.4) in the second case, in which the original Hilbert space $\mathcal{H}_{\mathrm{cft}} \otimes \mathcal{H}_{\mathrm{CFT}}$ is typically enlarged. If the messengers are scalar fields, $t_{a b}$ and $T_{a b}$ are still well defined in the new Hilbert space. But if they mediate gauge interactions these operators will not be gauge invariant any more. A simple fix would be to remove from the old $t_{a b}$ and $T_{a b}$ all the fields that participate in this gauge

\footnotetext{
${ }^{7}$ The relation between scaling dimension and mass for spin- 2 states is $m_{g}^{2} l_{\text {AdS }}^{2}=\Delta(\Delta-d)$. In warped compactifications both the AdS radius and the mass vary and only their product, which stays fixed, has invariant meaning. For the case at hand if the two AdS radii are different we have $m_{g}^{2} l_{\text {AdS }}^{2}=M_{g}^{2} L_{\text {AdS }}^{2}$.
} 
interaction. Our implicit assumption in what follows is that the linear combinations (3.4) can be defined, and are close (in the sense of operator norm) to the two lowest-lying spin-2 operators of the exact interacting theory.

Another important remark is also in order. In conventional holography one can only distinguish single- from multi-trace operators in the planar or zero-string-coupling $\left(g_{s}\right)$ limit. At any finite $g_{s}$ the two types of operators mix and eigenstates of the energy are linear combinations of operators of both types. What we mean, on the other hand, by 'double-trace' in this paper are products of an operator of $\mathrm{cft}_{d}$ with one of $\mathrm{CFT}_{d}$. Such operators can be clearly distinguished when the two conformal theories don't talk, for any values of the string coupling constants. A more appropriate name would have been 'bridging' operators, but we will keep refering to them as 'double-trace.'

\subsection{No multitrace for $N_{Q}>4$}

Before discussing other similarities or differences, let us see if these two mechanisms are compatible with supersymmetry. In section 2 we ruled out, on general grounds, all cases with $D>5$ and also all cases with $N_{Q}>8$. So we need only consider $\mathcal{N} \leq 2$ supersymmetries in $\mathrm{AdS}_{5}$ and $\mathcal{N} \leq 4$ supersymmetries in $\mathrm{AdS}_{4}$, i.e. four-dimensional SCFTs with $\mathcal{N} \leq 2$ and three-dimensional SCFTs with $\mathcal{N} \leq 4$. Now gauging a non-anomalous global symmetry is always allowed if $N_{Q} \leq 8$ (the largest value that admits vector multiplets) and the gauging is marginal or relevant in $d=4$ or $d=3$. Thus the 'messenger mechanism' does not exclude more cases than those already ruled out.

The 'double-trace mechanism' is more constrained. The deforming operators must now be top components of multiplets that are tensor products of two elementary ones, one from each of the two decoupled theories. This condition is impossible to meet while preserving $N_{Q}>4$ supercharges as I will now explain.

Consider first $\mathcal{N}=4, d=3$. There are no marginal (Lorentz-invariant) deformations in this case, and the only relevant ones are (i) hypermultiplet masses and Fayet-Iliopoulos terms, or (ii) a universal mass deformation that resides in the multiplet of the conserved energy-momentum tensor [31]. None of these can couple two non-trivial decoupled theories. The standard mass deformations may only couple two free hypermultiplets. Likewise FayetIliopoulos terms can only couple two free twisted hypermultiplets, ${ }^{8}$ while the universal mass deformation flows to a gapped theory and is for us uninteresting.

The argument actually works also in the less supersymmetric $\mathcal{N}=3, d=3$ case. There are again no marginal deformations, and the only relevant ones are flavour masses that can only couple two free hypermultiplets. Finally we rule out $\mathcal{N}=2$ in $d=4$. Here the relevant and marginal deformations are of two kinds: (i) [Higgs-branch] flavour mass deformations, and (ii) [Coulomb-branch] deformations that reside in chiral multiplets with $\mathrm{U}(1)_{R}$ symmetry charge $2<r \leq 4[31,32]$. None of these is a product of $\mathcal{N}=2$

\footnotetext{
${ }^{8}$ These statements do not rely on a Lagrangian description. In the notation of ref. [11] the deformations reside in $B_{1}[0]^{(2,0)}$ multiplets (or in their mirrors). The 'multitrace' property requires them to be a tensor product of two $B_{1}[0]^{(1,0)}$ which are free hypermultiplets QED.
} 


\begin{tabular}{|c|c|c|c|c|}
\cline { 2 - 5 } \multicolumn{1}{c|}{} & Susy & Multitrace & Massless graviton & Stueckelberg \\
\hline \multirow{2}{*}{$\operatorname{AdS}_{5}$} & $\mathcal{N}=2$ & no & $A_{2} \bar{A}_{2}[0 ; 0]_{2}^{(0 ; 0)}$ & $B_{1} \bar{B}_{1}[0 ; 0]_{4}^{(4 ; 0)} \oplus\left(A_{2} \bar{B}_{1}[0 ; 0]_{3}^{(2 ; 2)} \oplus \mathrm{cc}\right)$ \\
\cline { 2 - 5 } & $\mathcal{N}=1$ & yes & $A_{1} \bar{A}_{1}[1 ; 1]_{3}^{(0)}$ & $L \bar{A}_{2}[1 ; 0]_{7 / 2}^{(1)} \oplus \mathrm{cc}$ \\
\hline \multirow{3}{*}{$\mathrm{AdS}_{4}$} & $\mathcal{N}=4$ & no & $A_{2}[0]_{1}^{0,0)}$ & $B_{1}[0]_{2}^{(2,2)}$ \\
\cline { 2 - 5 } & $\mathcal{N}=3$ & no & $A_{1}[1]_{3 / 2}^{(0)}$ & $A_{2}[0]_{2}^{(2)}$ \\
\cline { 2 - 5 } & $\mathcal{N}=2$ & yes & $A_{1} \bar{A}_{1}[2]_{2}^{(0)}$ & $L \bar{A}_{1}[1]_{5 / 2}^{(1)} \oplus \mathrm{cc}$ \\
\cline { 2 - 5 } & $\mathcal{N}=1$ & yes & $A_{1}[3]_{5 / 2}$ & $L[2]_{3}$ \\
\hline
\end{tabular}

Table 2. The AdS supergravities for which the graviton multiplet can obtain a mass by gauging a global symmetry of the dual SCFT. The alternative 'multi-trace mechanism' is only possible for $N_{Q} \leq 4$ supercharges. The superconformal algebras are $\mathfrak{s u}(2,2 \mid \mathcal{N})$ in $d=4$ and $\mathfrak{o s p}(\mathcal{N} \mid 4)$ in $d=3$. The two right-most columns list the massless graviton and the Stueckelberg multiplets in the notation of ref. [11]. The massive graviton multiplets contain $2^{N_{Q}-1}$ bosonic and $2^{N_{Q}-1}$ fermionic states.

multiplets except in the trivial case of free fields. ${ }^{9}$ The marginal Coulomb-branch operators, in particular, are all believed to be gauge-coupling and theta-angle deformations. I will comment more on these in the following section.

The cases $\mathcal{N}=2, d=3$ and $\mathcal{N}=1, d=4$ are not ruled out as is easily shown by examples. The marginal or relevant deformations for $\mathcal{N}=1, d=4$ are superpotential deformations in chiral multiplets with $\mathrm{U}(1)_{R}$ charge $2 \geq r>\frac{2}{3}$. One can form a marginal deformation $(r=2)$ from the product of two chiral multiplets with $r=1$. A famous example is the Klebanov-Witten theory [33] in which the bifundamental chiral fields have $R$ charge $r=\frac{1}{2}$, so there are gauge-invariant operators with $r=1$. The product of two such operators can be used to deform two previously decoupled Klebanov-Witten theories. The case $\mathcal{N}=2, d=3$ is even simpler, since quartic superpotentials are classically marginal. They can be used to make two decoupled theories interact. For instance $\mathcal{N}=4$ SCFTs have a plethora of marginal $\mathcal{N}=2$ deformations of this kind (see e.g. [34, 35]).

These conclusions are summarized in table 2. Also listed in this table are the multiplets that provide the extra polarization states of a massive supergraviton in all the cases where Higgsing is a priori allowed. Consistency requires that such Stueckelberg multiplets should be available whenever two decoupled theories are made to interact. We will now see that this is indeed always the case.

\subsection{Stueckelberg multiplets}

Since the reader may not be familiar with the representation-theoretic notation of ref. [11], let me discuss the case of $\mathcal{N}=1$ supersymmetry in $d=4$ in more familiar language. In this case the conserved and traceless energy-momentum tensor sits in a real vector superfield

\footnotetext{
${ }^{9}$ The statement does not again rely on a Lagrangian description. The two types of operators reside in multiplets (i) $B_{1} \bar{B}_{1}[0]^{(2 ; 0)}$ or (ii) $L \bar{B}_{1}[0]^{(0 ; r)}$ where the superscript gives the $\mathrm{SU}(2)_{R} \times \mathrm{U}(1)_{R}$ quantum numbers, and $2<r \leq 4$ [11]. These operators can only be tensor products of the multiplets $B_{1} \bar{B}_{1}[0]^{(1 ; 0)}$ or $A_{2} \bar{B}_{1}[0 ; 0]^{(0 ; 2)}$ which describe, respectively, a free hypermultiplet and a free vector multiplet.
} 
$R_{\alpha \dot{\alpha}}$, subject to the conditions $\bar{D}^{\dot{\alpha}} R_{\alpha \dot{\alpha}}=D^{\alpha} R_{\alpha \dot{\alpha}}=0$. This superfield includes in addition to $T_{a b}$ the conserved $R$-symmetry current $R_{a}$ (see [36] for a nice discussion of the energymomentum superfield for $\mathcal{N}=1$ in $d=4$ ). In the notation of ref. [11] this is the multiplet $A_{1} \bar{A}_{1}[1 ; 1]_{3}^{(0)}$. The conditions $\bar{D}^{\dot{\alpha}} R_{\alpha \dot{\alpha}}=D^{\alpha} R_{\alpha \dot{\alpha}}=0$ imply indeed that null states appear at level 1 as indicated by the subscripts of $A$.

Initially, each of the two decoupled SCFTs has its own conserved $R$ superfield. After coupling these $R$-superfields mix linearly like their spin-2 components in eq. (3.4), so that the two linear combinations now obey the conditions

$$
D^{\alpha} \mathcal{R}_{\alpha \dot{\alpha}}=\bar{D}^{\dot{\alpha}} \mathcal{R}_{\alpha \dot{\alpha}}=0, \quad \bar{D}^{\dot{\alpha}} \mathcal{R}_{\alpha \dot{\alpha}}^{\prime}=V_{\alpha} \quad \text { and } \quad D^{\alpha} \mathcal{R}_{\alpha \dot{\alpha}}^{\prime}=-\bar{V}_{\dot{\alpha}} .
$$

Here $\mathcal{R}_{\alpha \dot{\alpha}}$ is the total $R$ superfield which remains conserved, and $V_{\alpha}$ is the Stueckelberg superfield which obeys the second-order condition $\bar{D}^{2} V_{\alpha}=0$ and renders (in the dual supergravity) the second graviton massive. The $L \bar{A}_{2}[1 ; 0]^{(1)}$ representation of $\mathfrak{s u}(2,2 \mid 1)$ in table 2, with null states at level 2 , corresponds precisely to the multiplet $V_{\alpha}$. Note that the familiar Ferrara-Zumino superfield [37] is also defined as a pair $\left(R_{\alpha \dot{\alpha}}, V_{\alpha}\right)$ but with $V_{\alpha}$ further constrained to ensure energy-momentum conservation [36]. Here, after coupling the two theories, none of the currents in the superfield $\mathcal{R}_{\alpha \dot{\alpha}}^{\prime}$ are conserved.

Consider now the marginal superpotential $W=\phi \Phi$ with $\phi$ a chiral superfield of $\mathrm{cft}_{4}, \Phi$ a chiral superfield of $\mathrm{CFT}_{4}$, and the sum of $R$ charges equal to 2 . It is easy to see that the spinor superfield $V_{\alpha}:=\phi D_{\alpha} \Phi-\Phi D_{\alpha} \phi$ has the desired properties to be the Stueckelberg field. It has the right scaling dimension and $R$ charge, and obeys the second-order condition $\bar{D}^{2} V_{\alpha}=0$. The story generalizes to all cases in table 2 that admit marginal doubletrace deformations. Let the same symbol $\phi$ denote the superconformal representation that includes (in the sense of the operator-state correspondence) the chiral superfield and all of its derivatives. One finds in all $N_{Q} \leq 4$ cases the schematic decomposition

$$
\phi \otimes \Phi=\text { deformation } \oplus \text { Stueckelberg } \oplus \cdots
$$

showing that when a marginal double-trace deformation is available the Stueckelberg field is automatically in place. ${ }^{10}$

What about $N_{Q}>4$ and the messenger mechanism? Consider $\mathcal{N}=2$ in $d=4$. The basic ingredients for gauging a global symmetry are conserved currents of hypermultiplet global symmetries and free vector multiplets. These correspond, respectively, to the two unitary representations $B_{1} \bar{B}_{1}[0 ; 0]_{2}^{(2 ; 0)}$ and $A_{2} \bar{B}_{1}[0 ; 0]_{1}^{(0,2)}$ of $\mathfrak{s u}(2,2 \mid 2)$ [11]. Tensor products of these representations give precisely the Stueckelberg multiplets in table $2 .^{11}$

Things work out similarly for $\mathcal{N}=4$ in $d=3$. One difference is that a free vector multiplet has an associated topological current $\epsilon^{a b c} F_{b c}$, so the appropriate superconformal

\footnotetext{
${ }^{10}$ For $\mathcal{N}=1, d=3$ the Stueckelberg superfield is a long multiplet, another manifestation of the fact that marginal deformations are in this case accidental. In the dual $\mathcal{N}=1 \mathrm{AdS}_{4}$ supergravity this multiplet has six bosonic degrees of freedom giving (with the massless graviton) a massive spin 2 and a massive spin 1.

${ }^{11}$ The only other way to obtain these Stueckelberg multiplets as tensor products is from $B_{1} \bar{B}_{1}[0 ; 0]_{1}^{(1 ; 0)}$ and $A_{2} \bar{B}_{1}[1 ; 0]_{2}^{(1,2)}$ which correspond, respectively, to a free hypermultiplet and an extra conserved supercurrent multiplet [11]. Since the latter is not usually available in the decoupled SCFTs, gauging a global symmetry is the only generic way to couple weakly two non-trivial $d=4$ SCFTs while respecting $\mathcal{N}=2$ superconformal invariance.
} 
representation is that of a conserved-current multiplet $B_{1}[0]_{1}^{(0 ; 2)}$. Its product with a [Higgsbranch] conserved current $B_{1}[0]_{1}^{(2 ; 0)}$ gives precisely the Stueckelberg superfield of table 2 . This shows that the Stueckelberg fields for Higgsing the supergraviton are automatically in place also for the 'gauging mechanism'.

Note that the Stueckelberg multiplet can be thought of intuitively as the superspace reparametrization needed to map one Universe to the other.

\section{On massive AdS supergravities}

The microscopic embedding of refs. $[7,8]$ and the discussion of the previous section suggest the existence of effective theories of a massive supergraviton with $\mathcal{N} \leq 2$ supersymmetries in five dimensions, and with $\mathcal{N} \leq 4$ in four dimensions. In the maximally-supersymmetric allowed cases the massive supergraviton contains 128 bosonic and 128 fermionic fields, which split into those of a massless multiplet (24 bosons and as many fermions in $D=5$, and 16 in $D=4$ ) and a Stueckelberg multiplet (respectively 104 and 112 bosons and as many fermions). A possibly significant coincidence is that the total number of fields in these massive-graviton multiplets is the same as for the familar maximal supergravities in $D=4,5$ dimensions. ${ }^{12}$

Constructing these effective massive supergravities is a nice technical challenge. Massive linearized actions for $\mathcal{N}=1$ in flat $D=4$ dimensions have been constructed in refs. [38-40] but little is known for extended supersymmetry and for AdS backgrounds. In the case $D=4 \mathcal{N}=4$ one could in principle compute the effective action from the string theory embedding $[7,8]$, but in practice this is a formidable task. The direct construction is a more realistic project. Here I assume that the putative supergravities (for all cases of table 2) do exist, and make some comments about their validity range.

\subsection{Breakdown and a distance conjecture}

The range of validity of the effective theories is a central issue in the bottom-up approach to massive gravity. The question is qualitatively different in the Minkowski and Anti-de Sitter backgrounds because the scalar mode of the graviton behaves differently in the two cases. In the $\operatorname{AdS}_{D}$ case of interest to us here, the largest energy scale $\Lambda_{\star}$ at which all effective theories are believed to break down is [10]

$$
\Lambda_{\star}^{D+2} \sim \frac{m_{g}^{2} m_{\mathrm{Pl}}^{D-2}}{l_{\mathrm{AdS}}^{2}} .
$$

This limit follows from a power-counting argument for the optimal non-renormalizable action of the Stueckelberg field (which comes entirely from the non-linear completion of the mass term). The argument is robust enough to [most likely] survive supersymmetric completions. In flat spacetime it was indeed shown [41, 42] that no finite number of spin $<2$ fields can push the corresponding breakdown scale to higher energy.

One may convert this $\Lambda_{\star}$ scale to a cutoff on scaling dimensions in the conformal theory by multiplying both sides of (4.1) with $l_{\mathrm{AdS}}^{D+2}$. For spin- 2 fields whose mass-dimension

\footnotetext{
${ }^{12}$ I thank Pierre Fayet for this comment.
} 
relation is $\Delta_{*}\left(\Delta_{*}-D+1\right)=\Lambda_{\star}^{2} l_{\mathrm{AdS}}^{2}$, this gives

$$
\Delta_{*} \sim \begin{cases}(D-1)+\left(\epsilon_{g} c\right)^{\frac{2}{D+2}} & \text { if } \epsilon_{g} c \ll 1, \\ \left(\epsilon_{g} c\right)^{\frac{1}{D+2}} & \text { if } \epsilon_{g} c \gg 1\end{cases}
$$

The meaning of the above estimate is as follows. In any CFT with holographic dual and in which the lowest spin-2 operator has anomalous dimension $\epsilon_{g} \ll 1$, new operators of spin $\geq 2$ will enter at scaling dimensions $\sim \Delta_{\star}$, where $c$ is the central charge of the CFT.

Actually only the upper branch of the bound (4.2) is relevant to our discussion of massive gravity. This is because towers of spin-2 excitations with scaling-dimension spacings $\sim O(1)$ are anyway a generic feature of all holographic models. Such towers are inevitable in theories with $N_{Q}>2$ supercharges which have continuous $R$ symmetries. The towers in this case correspond to Kaluza-Klein excitations along the compact submanifold whose isometries realize the continuous $R$ symmetry. In addition multitrace operators of spin-2 are generic at $\Delta \sim O(1)$ and hard to disentangle from single-trace operators. ${ }^{13}$

We assume then that $\epsilon_{g} c \ll 1$, i.e. that the cutoff $\Lambda_{\star}$ is much below the characteristic AdS scale. In this case the bound (4.2) predicts a condensation of spin-2 modes, so that the limit of zero graviton mass should be a decompactification limit at infinite distance. One can formulate this as a 'distance conjecture' for massive spin-2 particles in AdS. ${ }^{14}$

Massive-AdS-graviton conjecture: if the lowest-lying graviton mass $\left(m_{g}\right)$ varies continuously in a family of $A d S_{D}$ vacua of string theory, then $m_{g}=0$ is at infinite distance in moduli space. Furthermore, approaching this point brings down a tower of spin $\geq 2$ excitations with mass spacings vanishing as $\Lambda_{\star} \sim m_{g}^{2 / D+2} m_{\mathrm{Pl}}^{D-2 / D+2}$ in units of the $A d S$ radius, or faster.

Both statements of this conjecture can be verified in the microscopic embedding of $\mathcal{N}=4$ $\mathrm{AdS}_{4}$ massive gravity, as I will explain in the next subsection. In the case of $\mathcal{N}=2 \mathrm{AdS}_{5}$ the parameter controlling the graviton mass is typically a marginal gauge coupling, $\tau$, of the $\mathrm{SCFT}_{4}$. The decoupling limit $\tau=\infty$ is at infinite distance in simple examples, but it would be interesting to give a general proof. Furthermore, characterizing the decoupling limit more precisely should allow to reinforce or invalidate the second part of the conjecture.

\subsection{Janus throat and the $\Lambda_{*}$ scale}

The string-theory embedding of refs. [7, 8] is dual to a pair of $\mathcal{N}=4, d=3$ superconformal theories coupled by gauging a common 'small' flavour symmetry, $\mathrm{U}(n)$ with $n^{2} \ll c, C$. Since gauging is a relevant deformation in three dimensions, we let it flow to an infrared fixed point. This is in general a strongly-coupled fixed point, yet the effect on the low-lying

\footnotetext{
${ }^{13}$ This is sometimes refered to as the AdS 'scale separation problem'. The problem could be in principle avoided for $\mathcal{N}=1$ in $\mathrm{AdS}_{4}$, the case where marginal deformations need fine-tuning and are accidental. Even in this case, however, one expects towers of higher-spin multi-particle states with spacings $\sim O(1)$, because of the attractive nature of gravity and the focussing effect of AdS spacetime.

${ }^{14}$ See [43-45] for recent discussions of swampland conjectures involving spin-2 fields in flat spacetime.
} 


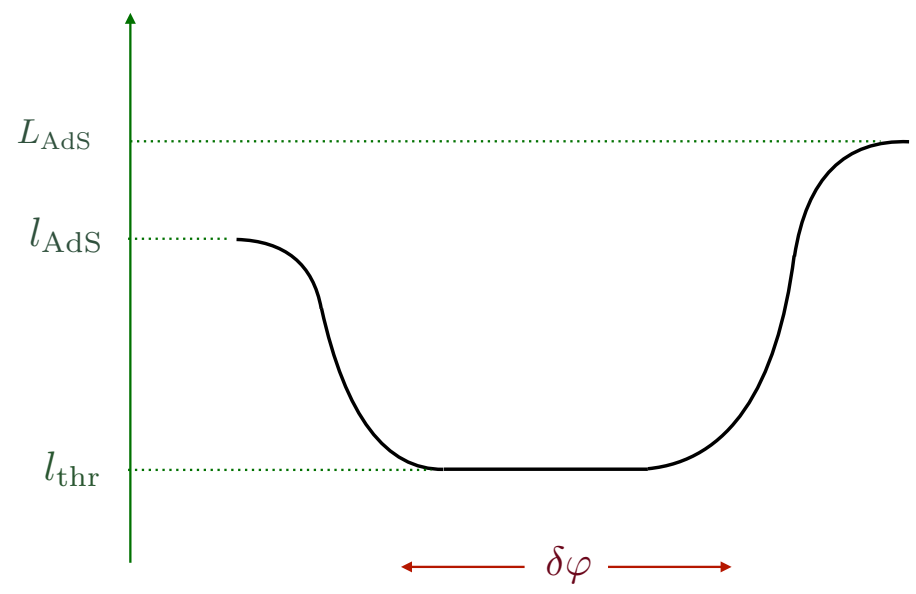

Figure 1. Schematic plot of the Janus metric $d s_{\mathrm{Janus}}^{2} \simeq l_{\mathrm{thr}}^{2} d x^{2}+\ell(x)^{2} d s_{\mathrm{AdS}_{4}}^{2}$. The horizontal and vertical axes parametrize, respectively, the coordinate $x$ and the radius $\ell(x)$ of the $\operatorname{AdS}_{4}$ fiber. The relevant parameters are the asymptotic left and right radii, $l_{\mathrm{AdS}}$ and $L_{\mathrm{AdS}}$, the radius $l_{\mathrm{thr}}$ of the throat, and the size of its flat region $\delta x \sim \delta \varphi$ over which the string-theory dilaton varies linearly.

spin-2 spectrum can be arbitrarily small. Both the disjoint theories and the coupled one are SCFTs of the type conjectured by Gaiotto and Witten [46].

The corresponding type IIB solutions were derived for this class of theories in refs. [47, 48]. In the limit of interest their key feature is a Janus throat carrying $n$ units of five-form flux, and capped off at both sides by large compact six-manifolds $\mathcal{M}_{6}$ and $\mathcal{M}_{6}^{\prime}$ (see the figure). The (warped) compactification $\mathrm{AdS}_{4} \times_{w} \mathcal{M}_{6}$ with AdS radius $l_{\mathrm{AdS}}$ is dual to $\mathrm{cft}_{3}$, and $\mathrm{CFT}_{3}$ is likewise dual to $\mathrm{AdS}_{4} \times_{w} \mathcal{M}_{6}^{\prime}$ with AdS radius $L_{\mathrm{AdS}}$. One can think of the throat region as the hologram of the $\mathrm{U}(n)$ messenger degrees of freedom connecting the two theories.

The supersymmetric Janus solution was found in ref. [49]. It extrapolates between two asymptotic $\mathrm{AdS}_{5} \times \mathrm{S}^{5}$ regions where the metric approaches $d s^{2} \simeq l_{\mathrm{thr}}^{2}\left(d x^{2}+\cosh ^{2} x d s_{\mathrm{AdS}_{4}}^{2}\right)$, and the string-theory dilaton asymptotes to constant values $\varphi$ and $\varphi^{\prime}$. The dilaton varies linearly in a region of size $\delta x \simeq \delta \varphi=\left|\varphi-\varphi^{\prime}\right|$ at the bottom of the throat. For $\delta \varphi=0$ the solution reduces to the standard $\operatorname{AdS}_{5} \times S^{5}$ geometry with radius $l_{\text {thr }} \sim n^{1 / 4}$. In our setup the two asymptotic regions are capped off at some large values of $x$ on the left and the right. One can think of these caps as 'end of the world' branes in a Randall-Sundrum compactification [18-20]. However, not only these branes are not thin, but on the contrary they occupy most of spacetime.

The full string theory solution has a large number of parameters describing the quivers of $\mathrm{cft}_{3}$ and $\mathrm{CFT}_{3}$, but only five are relevant for the description of the Janus bridge: the three radii $\left(l_{\mathrm{thr}} \ll l_{\mathrm{AdS}}, L_{\mathrm{AdS}}\right)$ and the two asymptotic values of the dilaton. Equivalently, these latter can be replaced by the effective four-dimensional Planck masses which, in units of the string scale, behave parametrically as

$$
m_{\text {Planck }}^{2} \sim l_{\text {AdS }}^{6} e^{2 \varphi} \quad \text { and } \quad M_{\text {Planck }}^{2} \sim L_{\text {AdS }}^{6} e^{2 \varphi^{\prime}} .
$$

On the field theory side the five parameters are $n, c, C$ and the two trilinear couplings of 
the cft and CFT energy-momentum tensors, $\langle t t t\rangle$ and $\langle T T T\rangle$. In the specific realization of ref. [8] the dilaton parameters are ratios of electric-to-magnetic flavour-group ranks in the two quiver theories, or equivalently ratios of D5-to NS5 brane charges in the IIB solutions. We wont need the precise relation between $\varphi, \varphi^{\prime}$ and the parameters of $\mathrm{cft}_{3}$ and $\mathrm{CFT}_{3}$, only the fact that these are rational parameters that can be varied independently from all others.

Let us now fix the left theory $\left(\mathrm{cft}_{3}\right)$ on which our privileged observer lives, and vary the parameters of the right theory $\left(\mathrm{CFT}_{3}\right)$. To keep the discussion simple, I take rightaway the limit $L_{\mathrm{AdS}} \rightarrow \infty$ so that the right-hand-side of the solution asymptotes to $\operatorname{AdS}_{5} / \mathbb{Z}_{2} \times \mathrm{S}^{5}$. This solution is dual to a familiar setup, namely $\mathcal{N}=4, d=4$ super Yang-Mills interacting with a strongly-coupled theory, $\mathrm{cft}_{3}$, on the boundary of space. The $4 d$ Yang-Mills theory has gauge group $\mathrm{U}(n)$ with $n^{2} \ll c$, so most degrees of freedom live on the boundary, not in the bulk. ${ }^{15}$ Its only other parameter is the four-dimensional Yang-Mills coupling constant that varies continuously with the dilaton, $g_{\mathrm{YM}}^{2} \sim e^{-\varphi^{\prime}}$.

One expects intuitively that the leaking-out of energy from $\mathrm{cft}_{3}$ can be suppressed in two different ways: (a) by the scarcity of bulk degrees of freedom $\left(n^{2} \ll c\right)$, and (b) in the decoupling limit $g_{\mathrm{YM}} \rightarrow 0$, i.e. $\varphi^{\prime} \rightarrow \pm \infty$ (the two signs are related by S-duality). Both factors should contribute to make the mass of the graviton parametrically small, as is indeed confirmed by the calculation of ref. [8].

The basic idea behind this calculation is that the wavefunction of the lowest-lying spin2 mode vanishes at $x \rightarrow \infty$ and approaches exponentially fast a value, $\psi_{0}$, at the other end of the Janus throat. Here $\psi_{0}$ is the constant wavefunction of the massless graviton in the $\mathrm{AdS}_{4} \times_{w} \mathcal{M}_{6}$ vacuum dual to the boundary $\mathrm{cft}_{3}$. This is a non-normalizable mode in the full Janus background, but it becomes normalizable thanks to the cutoff in the $x \rightarrow-\infty$ region. Calculating the mass gives [8]

$$
\epsilon_{g} \simeq \underbrace{\frac{n^{2}}{4 \pi^{4}} \frac{\tanh ^{3} \delta \varphi}{[\delta \varphi-\tanh \delta \varphi]}}_{\lambda_{\mathrm{eff}}^{2}} \times \frac{1}{c} .
$$

This is indeed proportional both to $n^{2} / c$, and to $\delta \varphi^{-1}$ for large $\delta \varphi$, i.e. to the two suppression factors advertized above.

We may now return to the question of the breakdown scale $\Lambda_{\star}$. Let us write $\epsilon_{g} c:=\lambda_{\text {eff }}^{2}$ by analogy with the result of conformal perturbation theory, eq. (3.5), for CFTs coupled by a double-trace deformation [15]. We are interested in the limit $\lambda_{\text {eff }} \rightarrow 0$ in which the graviton becomes massless. We could try to take this limit in two ways:

- By taking $n \rightarrow 0$. This is possible in supergravity where $n \sim l_{\text {thr }}^{4}$ is a continuous parameter, but in string theory $n$ [the D3-brane charge of the throat] is quantized.

- By taking $\delta \varphi \rightarrow \infty$. This is at infinite distance in moduli space, in agreement with the first part of our conjecture. In this limit the Janus throat develops a flat region of

\footnotetext{
${ }^{15}$ 'Bulk' and 'boundary' here refer to the conformal field theory side of the duality. On the gravity side they correspond to the $\mathrm{AdS}_{5} / \mathbb{Z}_{2}$ spacetime and the thick-cap $\mathrm{AdS}_{4}$ brane.
} 
invariant length $\sim l_{\text {thr }} \delta \varphi$, bringing down spin-2 Kaluza-Klein excitations with mass spacings $\sim\left(l_{\mathrm{thr}} \delta \varphi\right)^{-1} .{ }^{16}$ The dual tower of spin-2 operators on the field theory side would thus have anomalous scaling dimensions $\sim(\delta \varphi)^{-2} \ll(\delta \varphi)^{-1 / 3} \sim \Delta_{*}-3$, in agreement with the second part of the massive-graviton conjecture.

It is striking that the full string-theory embedding of the 'bridge' is instrumental for reaching these conclusions. In ten-dimensional supergravity the limit of vanishing Janus radius is smooth, leaving behind harmless coordinate singularities [47, 51]. Without the string theoretic quantization of $l_{\mathrm{thr}}$ this would be in tension with the breakdown of the effective massive-gravity theory. From the CFT side on the other hand, although it might be sometimes clear that the decoupling limit is at infinite distance, the nature of this limit can be obscure. It is important in this regard to stress that, in the above example, the condensing spin-2 states on the gravity side are in long unprotected multiplets. Even though the entire setup has $\mathcal{N}=4$ supersymmetry, the singularity of the effective gravitational theory has its origin in the non-BPS sector.

These results raise two immediate questions. First, can the above analysis be extended to five-dimensional $\mathcal{N}=2$ supergravity, the other maximally-supersymmetric case among the possibilities of table 2? The menagerie of four-dimensional $\mathcal{N}=2$ SCFTs has been greatly extended by the construction of class-S theories [52], and much is known about their moduli spaces and their supergravity duals [53]. An analysis similar to the one presented here for $\mathcal{N}=4 \mathrm{SCFT}_{3}$, is possibly within reach. ${ }^{17}$

The second question concerns double-trace deformations. The standard recipe maps them to modified boundary conditions on the gravity side $[12,13]$. But this is a recipe in the effective $D$-dimensional supergravity and its lift to ten dimensions, let alone its embedding in the full string theory, is problematic. The lesson from our discussion in this paper is that the string-theoretic description of the 'double-trace bridge' may be much richer than hitherto imagined.

It is logically conceivable that the marginal double-trace modulus, $\lambda$, of the CFT is quantized, but there is no such precedent in string theory that I am aware of. The more plausible hypothesis is that, as was the case for the Janus throat, the $\lambda \rightarrow 0$ limit is at infinite distance and brings down a tower of light spin $\geq 2$ states. It might be possible to study this question with the conformal bootstrap.

\section{Acknowledgments}

I have benefited from discussions with Ali Chamseddine, Jean-Pierre Derendinger, Sergei Dubovsky, Marc Henneaux, Chris Hull, Ioannis Lavdas, Bruno Le Floch, George Pa-

\footnotetext{
${ }^{16}$ The spin-2 Kaluza-Klein spectrum in the Janus background can be computed analytically [50]. The result confirms the above heuristic argument.

${ }^{17}$ C.B and A. Tomasiello, work in progress. The superconformal index, a powerful tool for the study of $\mathcal{N}=2$ SCFTs, is unfortunately not adapted to our problem. The index is blind to long multiplets such as the conjectured unprotected towers of spin-2 states. Interestingly the authors of ref. [54] extracted the number of conserved energy-momentum tensors from the superconformal index of selected class-S theories. This is a good diagnostic for the number of non-interacting SCFTs, but not of the conjectured singular behavior under small marginal interactions.
} 
padopoulos, Massimo Porrati, Jan Troost, Alessandro Tomasiello and Misha Vassiliev. I am particularly indebted to Claudia de Rham and Andrew Tolley for a discussion that motivated the analysis in section 4. I am also grateful to the String Theory group at Imperial College for hospitality during part of this work.

Open Access. This article is distributed under the terms of the Creative Commons Attribution License (CC-BY 4.0), which permits any use, distribution and reproduction in any medium, provided the original author(s) and source are credited.

\section{References}

[1] K. Hinterbichler, Theoretical aspects of massive gravity, Rev. Mod. Phys. 84 (2012) 671 [arXiv:1105.3735] [INSPIRE].

[2] C. de Rham, Massive gravity, Living Rev. Rel. 17 (2014) 7 [arXiv:1401.4173] [INSPIRE].

[3] A. Schmidt-May and M. von Strauss, Recent developments in bimetric theory, J. Phys. A 49 (2016) 183001 [arXiv:1512.00021] [INSPIRE].

[4] LIGO ScIentific, VIRGO collaboration, GW170104: observation of a 50-solar-mass binary black hole coalescence at redshift 0.2, Phys. Rev. Lett. 118 (2017) 221101 [Erratum ibid. 121 (2018) 129901] [arXiv:1706.01812] [INSPIRE].

[5] C. de Rham, J.T. Deskins, A.J. Tolley and S.-Y. Zhou, Graviton mass bounds, Rev. Mod. Phys. 89 (2017) 025004 [arXiv: 1606.08462] [INSPIRE].

[6] C.M. Will, Solar system versus gravitational-wave bounds on the graviton mass, Class. Quant. Grav. 35 (2018) 17LT01 [arXiv:1805.10523] [INSPIRE].

[7] C. Bachas and I. Lavdas, Quantum gates to other universes, Fortsch. Phys. 66 (2018) 1700096 [arXiv: 1711.11372 ] [INSPIRE].

[8] C. Bachas and I. Lavdas, Massive Anti-de Sitter gravity from string theory, JHEP 11 (2018) 003 [arXiv: 1807.00591] [INSPIRE].

[9] N. Arkani-Hamed, H. Georgi and M.D. Schwartz, Effective field theory for massive gravitons and gravity in theory space, Annals Phys. 305 (2003) 96 [hep-th/0210184] [INSPIRE].

[10] C. de Rham, A.J. Tolley and S.-Y. Zhou, The $\Lambda_{2}$ limit of massive gravity, JHEP 04 (2016) 188 [arXiv: 1602.03721] [INSPIRE].

[11] C. Cordova, T.T. Dumitrescu and K. Intriligator, Multiplets of superconformal symmetry in diverse dimensions, JHEP 03 (2019) 163 [arXiv: 1612.00809] [INSPIRE].

[12] E. Witten, Multitrace operators, boundary conditions and AdS/CFT correspondence, hep-th/0112258 [INSPIRE].

[13] M. Berkooz, A. Sever and A. Shomer, 'Double trace' deformations, boundary conditions and space-time singularities, JHEP 05 (2002) 034 [hep-th/0112264] [INSPIRE].

[14] E. Kiritsis, Product CFTs, gravitational cloning, massive gravitons and the space of gravitational duals, JHEP 11 (2006) 049 [hep-th/0608088] [INSPIRE].

[15] O. Aharony, A.B. Clark and A. Karch, The CFT/AdS correspondence, massive gravitons and a connectivity index conjecture, Phys. Rev. D 74 (2006) 086006 [hep-th/0608089] [INSPIRE]. 
[16] E. Kiritsis and V. Niarchos, Interacting string multi-verses and holographic instabilities of massive gravity, Nucl. Phys. B 812 (2009) 488 [arXiv:0808.3410] [INSPIRE].

[17] M. Porrati, Higgs phenomenon for 4 - D gravity in anti-de Sitter space, JHEP 04 (2002) 058 [hep-th/0112166] [INSPIRE].

[18] L. Randall and R. Sundrum, A large mass hierarchy from a small extra dimension, Phys. Rev. Lett. 83 (1999) 3370 [hep-ph/9905221] [INSPIRE].

[19] L. Randall and R. Sundrum, An alternative to compactification, Phys. Rev. Lett. 83 (1999) 4690 [hep-th/9906064] [INSPIRE].

[20] A. Karch and L. Randall, Locally localized gravity, JHEP 05 (2001) 008 [hep-th/0011156] [INSPIRE].

[21] S. Ferrara, A. Kehagias and D. Lüst, Bimetric, conformal supergravity and its superstring embedding, JHEP 05 (2019) 100 [arXiv: 1810.08147] [INSPIRE].

[22] C. de Rham and G. Gabadadze, Generalization of the Fierz-Pauli action, Phys. Rev. D 82 (2010) 044020 [arXiv: 1007.0443] [INSPIRE].

[23] C. de Rham, G. Gabadadze and A.J. Tolley, Resummation of massive gravity, Phys. Rev. Lett. 106 (2011) 231101 [arXiv:1011.1232] [INSPIRE].

[24] S.F. Hassan and R.A. Rosen, On non-linear actions for massive gravity, JHEP 07 (2011) 009 [arXiv: 1103.6055] [INSPIRE].

[25] S.F. Hassan and R.A. Rosen, Resolving the ghost problem in non-linear massive gravity, Phys. Rev. Lett. 108 (2012) 041101 [arXiv:1106.3344] [INSPIRE].

[26] A.H. Chamseddine and V. Mukhanov, Hidden ghost in massive gravity, JHEP 03 (2013) 092 [arXiv: 1302.4367] [INSPIRE].

[27] S. Deser, K. Izumi, Y.C. Ong and A. Waldron, Problems of massive gravities, Mod. Phys. Lett. A 30 (2015) 1540006 [arXiv:1410.2289] [InSPIRE].

[28] W. Nahm, Supersymmetries and their representations, Nucl. Phys. B 135 (1978) 149 [INSPIRE].

[29] E.A. Bergshoeff, O. Hohm and P.K. Townsend, Massive gravity in three dimensions, Phys. Rev. Lett. 102 (2009) 201301 [arXiv:0901.1766] [INSPIRE].

[30] R. Andringa et al., Massive 3D supergravity, Class. Quant. Grav. 27 (2010) 025010 [arXiv:0907.4658] [INSPIRE].

[31] C. Cordova, T.T. Dumitrescu and K. Intriligator, Deformations of superconformal theories, JHEP 11 (2016) 135 [arXiv:1602.01217] [INSPIRE].

[32] P. Argyres, M. Lotito, Y. Lü and M. Martone, Geometric constraints on the space of $\mathcal{N}=2$ SCFTs. Part I: physical constraints on relevant deformations, JHEP 02 (2018) 001 [arXiv: 1505.04814] [INSPIRE].

[33] I.R. Klebanov and E. Witten, Superconformal field theory on three-branes at a Calabi-Yau singularity, Nucl. Phys. B 536 (1998) 199 [hep-th/9807080] [InSPIRE].

[34] C. Bachas, M. Bianchi and A. Hanany, $\mathcal{N}=2$ moduli of $A d S_{4}$ vacua: a fine-print study, JHEP 08 (2018) 100 [Erratum ibid. 10 (2018) 032] [arXiv:1711.06722] [INSPIRE].

[35] C. Bachas, I. Lavdas and B. Le Floch, Marginal deformations of $3 d N=4$ linear quiver theories, arXiv:1905.06297 [INSPIRE]. 
[36] J.P. Derendinger, Currents in supersymmetric field theories, PoS (PLANCK 2015) 034 [arXiv: 1609.00164].

[37] S. Ferrara and B. Zumino, Transformation properties of the supercurrent, Nucl. Phys. B 87 (1975) 207 [INSPIRE].

[38] Yu.M. Zinoviev, Massive spin two supermultiplets, hep-th/0206209 [INSPIRE].

[39] T. Gregoire, M.D. Schwartz and Y. Shadmi, Massive supergravity and deconstruction, JHEP 07 (2004) 029 [hep-th/0403224] [INSPIRE].

[40] N.A. Ondo and A.J. Tolley, Deconstructing supergravity: massive supermultiplets, JHEP 11 (2018) 082 [arXiv:1612.08752] [INSPIRE].

[41] C. de Rham, S. Melville, A.J. Tolley and S.-Y. Zhou, Positivity bounds for massive spin-1 and spin-2 fields, JHEP 03 (2019) 182 [arXiv:1804.10624] [INSPIRE].

[42] J. Bonifacio, K. Hinterbichler and R.A. Rosen, Constraints on a gravitational Higgs mechanism, arXiv: 1903.09643 [INSPIRE].

[43] D. Klaewer, D. Lüst and E. Palti, A spin-2 conjecture on the swampland, Fortsch. Phys. 67 (2019) 1800102 [arXiv: 1811.07908] [inSPIRE].

[44] C. De Rham, L. Heisenberg and A.J. Tolley, Spin-2 and the weak gravity conjecture, arXiv: 1812.01012 [INSPIRE].

[45] E. Palti, The swampland: introduction and review, 2019, arXiv:1903.06239 [INSPIRE].

[46] D. Gaiotto and E. Witten, S-duality of boundary conditions in $N=4$ super Yang-Mills theory, Adv. Theor. Math. Phys. 13 (2009) 721 [arXiv:0807.3720] [InSPIRE].

[47] B. Assel, C. Bachas, J. Estes and J. Gomis, Holographic duals of $D=3 N=4$ superconformal field theories, JHEP 08 (2011) 087 [arXiv:1106.4253] [INSPIRE].

[48] B. Assel, C. Bachas, J. Estes and J. Gomis, IIB duals of $D=3 N=4$ circular quivers, JHEP 12 (2012) 044 [arXiv:1210.2590] [INSPIRE].

[49] E. D'Hoker, J. Estes and M. Gutperle, Ten-dimensional supersymmetric Janus solutions, Nucl. Phys. B 757 (2006) 79 [hep-th/0603012] [INSPIRE].

[50] C. Bachas and J. Estes, Spin-2 spectrum of defect theories, JHEP 06 (2011) 005 [arXiv: 1103.2800] [INSPIRE].

[51] O. Aharony, L. Berdichevsky, M. Berkooz and I. Shamir, Near-horizon solutions for D3-branes ending on 5-branes, Phys. Rev. D 84 (2011) 126003 [arXiv:1106.1870] [INSPIRE].

[52] D. Gaiotto, $N=2$ dualities, JHEP 08 (2012) 034 [arXiv:0904.2715] [INSPIRE].

[53] D. Gaiotto and J. Maldacena, The gravity duals of $N=2$ superconformal field theories, JHEP 10 (2012) 189 [arXiv:0904.4466] [INSPIRE].

[54] J. Distler, B. Ergun and F. Yan, Product SCFTs in class-S, arXiv: 1711.04727 [INSPIRE]. 